\title{
Design and Comparative Evaluation of Visualization Onboarding Methods
}

\author{
Christina Stoiber \\ St. Poelten UAS, Austria \\ christia.stoiber@fhstp.ac.at \\ Holger Stitz \\ datavisyn $\mathrm{GmbH}$, Austria \\ holger.stitz@datavisyn.io
}

\author{
Conny Walchshofer \\ JKU Linz, Austria \\ conny.walchshofer@jku.at \\ Marc Streit \\ JKU Linz, Austria \\ marc.streit@jku.at
}

\author{
Florian Grassinger \\ St. Poelten UAS, Austria \\ florian.grassinger@fhstp.ac.at \\ Wolfgang Aigner
St. Poelten UAS, Austria \\ wolfgang.aigner@fhstp.ac.at
}

\begin{abstract}
Comprehending and exploring large and complex data is becoming increasingly important for a diverse population of users in a wide range of application domains. Visualization has proven to be well-suited in supporting this endeavor by tapping into the power of human visual perception. However, non-experts in the field of visual analysis often have difficulties in correctly reading and interpreting information from novel visualization idioms. Visualization onboarding can support novices in learning how to use new digital technologies. Therefore, we developed an interactive step-by-step guide and applied the method to four visualization techniques - a bar chart, a horizon graph, a change matrix, and a parallel coordinates plot. Results using Amazon Mechanical Turk workers show that there is a need for onboarding, especially for more complex visualization techniques. We further investigated the perception and rating of a scrollytelling and a video tutorial for the most unfamiliar visualization-the parallel coordinates plot. A comparison between the three onboarding methods indicates that participants appreciated the easy-to-understand examples, the precise wording of the onboarding messages in a step-by-step manner, and the introduction of interaction concepts by highlighting the most relevant information over all onboarding methods. The video tutorial supported the introduction of unknown interaction techniques best.
\end{abstract}

\section{CCS CONCEPTS}

- Human-centered computing $\rightarrow$ Visualization design and evaluation methods.

\section{KEYWORDS}

Visualization literacy, user onboarding, learning, visual analytics

\section{ACM Reference Format:}

Christina Stoiber, Conny Walchshofer, Florian Grassinger, Holger Stitz, Marc Streit, and Wolfgang Aigner. 2021. Design and Comparative Evaluation of Visualization Onboarding Methods. In The 14th International Symposium on Visual Information Communication and Interaction (VINCI '21), September

\section{c) (i) (-)}

This work is licensed under a Creative Commons

Attribution-NonCommercial-ShareAlike International 4.0 License.

VINCI '21, September 6-8, 2021, Potsdam, Germany

(c) 2021 Copyright held by the owner/author(s).

ACM ISBN 978-1-4503-8647-0/21/09.

https://doi.org/10.1145/3481549.3481558
6-8, 2021, Potsdam, Germany. ACM, New York, NY, USA, 5 pages. https: //doi.org/10.1145/3481549.3481558

\section{INTRODUCTION}

Visualization is a tool that has become indispensable not only in the context of science and business, but also in everyday life, such as data stories in newspapers, in books, or on the Internet. The amount of data and the associated complexity often exceed the capabilities of traditional business charts, such as bar charts, line charts, or pie charts. As a consequence, more advanced visual representations are needed to capture large and complex datasets.

Visual mapping is the process of assigning data variables to visual channels, which results in either a static or an interactive visual representation. This process is the central component of virtually all known conceptual models of visualization, such as the model by Card et al. [9] or van Wijk [49]. Understanding the process of visual mapping is a key component for correctly decoding both the visual representation and the underlying data. Furthermore, data analysis, filtering, and rendering steps of the visualization process influence the appearance of a visualization and need to be used and selected precisely. Especially for novice users, however, this can be difficult and may lead to wrong conclusions as well as erroneous insights into the data. Visualization onboarding methods aim to support end users in comprehending data visualizations and taking full advantage of the tools at hand [46]. So far, there has been little discussion about the design of onboarding methods for visualization types and visual analytics tools. A few onboarding methods exist in the literature using different strategies and educational theories, such as learning by doing [25], learning by analogy [41], scaffolding [4], or top-down and bottom-up teaching methods as well as active and passive learning types [48]. Nonetheless, much more research is needed to identify effective designs of onboarding methods and to understand users' behavior while using onboarding methods.

Therefore, we extended the current literature by assessing the need and active usage of visualization onboarding for four different visualization types (bar chart, horizon graph, change matrix, and parallel coordinates). We use textual instructions, divided into the three sections of Reading the Chart, Interacting with the Chart, and Using the Chart, combined with in-place annotations to onboard users to a visualization. Qualitative feedback on interaction behavior with the onboarding method was captured and assessed using Amazon Mechanical Turk (MTurk) workers. Based on the most unfamiliar and most complex visualization type, the parallel coordinates plot, we investigated differences in the perception and 
rating of three onboarding methods by analyzing feedback on a step-by-step guide, a scrollytelling tutorial, and a video tutorial with a voice-over. Thus, we further developed our onboarding methods by designing the scrollytelling tutorial and the video tutorial with voice-over for a parallel coordinates plot. In a second phase of the evaluation with MTurk workers, we investigated differences in the perception and rating of the three different onboarding methods.

\section{RELATED WORK}

We base the design of the onboarding user interfaces and instructional materials on insights and lessons learned from empirical research, as well as on the analysis of contextually relevant visualization platforms and websites [19, 39, 47].

A comparative study conducted by Kwon et al. [25] shows that participants who use an interactive guide or a video tutorial outperform participants who use a static tutorial or none at all. Moreover, using a direct interaction with the visualization (also known as active learning) [48] or a learning-by-doing approach [25] positively influences the visualization comprehension. We additionally investigated popular visualization platforms and websites to understand non-scientific explanation approaches for different visualization types. The graphic continuum [47] provides an overview and helps in choosing the appropriate design or visualization type. Furthermore, the visualization library Data Viz Catalogue [39] seeks to help users understand the encoding and building blocks of different visualization types. A decision tree provided by From Data to $V i z$ [19] helps to find an appropriate visualization type based on the input data. The catalogue offers definitions, variations, and the use of each visualization type in addition to potential issues that may arise during use and interpretation. However, it quickly became apparent that the exclusively textual descriptions and used datasets on these platforms are very abstract-which is particularly problematic for novice users. Furthermore, all these instructions are provided externally and decoupled of the particular visualization.

Opinions on the dataset to be used for onboarding tend to differ sharply when it comes to explaining visualization systems and the underlying data. On the one hand, literature proclaims abstract data to be more generalizable and transferable to a novel context [23]. On the other hand, more recent scientific work provides counterevidence. De Bock et al. [13], for instance, show a more successful knowledge transfer by using concrete examples. Similarly, when introducing new visualizations, it is recommended to use an easy and understandable dataset that can be assumed to be well-known to the general public [15, 24, 27] or to be domain-specific[45] and well-known to the end user.

\section{VISUALIZATION ONBOARDING METHOD}

Previous studies [21, 22] examined how people create, update, and explain their visualizations using only tangible building blocks. So far, there are no studies available exploring how persons explain a certain visualization type. To better understand the explanation strategies, we conducted preliminary interviews with 13 participants $(\mathrm{m}=8, \mathrm{f}=5$; Age: $M=30.23, S D=3.32)$. The participants indicated that they had moderate to high experience with visualizations and a background in computer science, accounting, and HCI. The systematic analysis outlined that the participants started by explaining the dataset and attributes before continuing with the visual encoding, e.g. "The first thing I would say is that the diagram explains how many hours of sunshine the city of Innsbruck had in previous years." (translated from German to English).

Based on these insights of the analysis of empirical studies, online platforms, and the interviews, we derived the following four key design requirements: (i) explain visual encoding and give examples on insights a user can generate (based on the interviews), (ii) use concrete examples [1,13], (iii) follow an active learning approach [48] and make use of an interactive guide, and (iv) video [25] as onboarding methods.

In the following section, we provide descriptions of our onboarding methods, describe their main components, provide rationales based on related literature, and elaborate on our design decisions.

\subsection{Interactive Step-by-Step Guide}

For the first approach, the visualization onboarding interface is divided into four sections, as illustrated in Figure 1. We start with an introductory part $\mathbf{A}$ that serves as a brief overview to explain the attributes within the dataset. We added navigation elements $\mathbf{B}$ to enable viewing all steps at a glance or exploring the instructions one after the other. To explain how to read, use, and interact with the chart, we provide interactive numbered textual descriptions $\mathbf{C}$ which enable the respective in-place annotations in the chart. Finally, D represents our interactive visualization.

Introduction A: Due to the importance of title elements and legends [5], we integrated an introductory sentence on top of our onboarding method to describe the basic idea of the visualization type and provide general information about the data.

Navigation B: The current or selected step is highlighted in the respective sections (reading, interacting, using) as well as in the visualization itself, while the other steps are greyed out. The user can click on the numbered textual description within the step-bystep guide or use the Next and Previous buttons on the top right (see Figure 1 B). As an alternative for those who do not need a step-by-step explanation on how to read, interact with, and use the chart, we provide a Show All toggle element to hide and show all hints at a glance.

Step-by-Step Messages C: Based on the explanatory sequences of our interview participants, the step-by-step guide incorporates textual descriptions on how to read, interact with, and use the chart. The first section contains information, e.g., about the visualizations' shape, axes, or color coding. The second section Interacting with the Chart emphasizes the applied interaction techniques, e.g, indicating how to re-order axes or filter attribute values. For the section Using the Chart, we provide three examples that make use of the low-level typology (identification, comparison, and summarization task) by Brehmer and Munzner [32]. The design is organized in numbered textual descriptions with highlighted attributes in combination with in-place annotations [50] (numbers and symbols) to indicate the connection between the highlighted text elements and the visual encoding. Inspired by different annotation designs by $\mathrm{Lu}$ [30], we use circular visual markers with numbers that relate to the selected step. To understand the visual encoding, we make use of data, guides, and marks from the Vega grammar [43] by highlighting the respective attributes in the onboarding message. Hence, in 

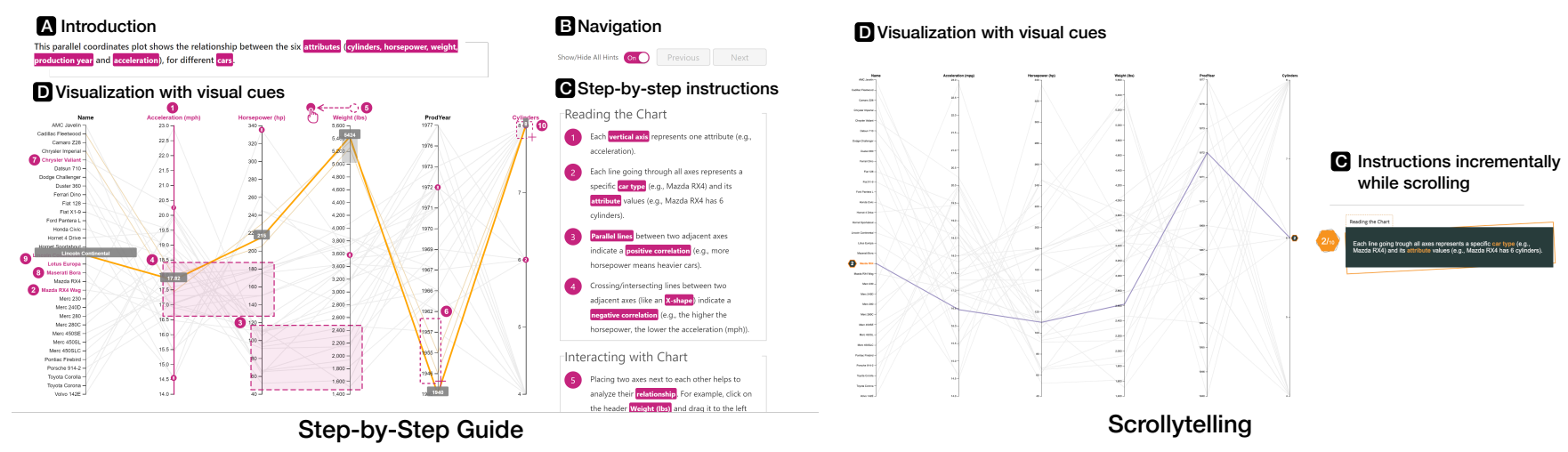

Figure 1: Two onboarding approaches are demonstrated using the example of a parallel coordinates plot-(left) a step-by-step guide and (right) a scrollytelling tutorial. They are based on textual descriptions and in-place annotations and consist of four parts: a brief textual introduction providing contextual information about the visualization $A$, navigational elements $B$ to go through the step-by-step instructions $\mathrm{C}$, and the visualization itself $\mathrm{D}$. We divide the textual descriptions into Reading the Chart, for explaining the visual encoding, Interacting with the Chart, for explaining the interaction concept, and Using the Chart, for providing exemplary insights. (The prototypes can be accessed here: https://onboarding-methods.netlify.app/)

each step, we accentuate words that are related to visual properties of the visualization, an encoding, a data transformation, a pattern, or a finding. Moreover, as seen in step 3 in Figure $1 \mathbf{C}$, we use rectangular elements to demonstrate how patterns (e.g., positive or negative correlations) can be read in parallel coordinates plots. In addition, for the interaction concepts, we introduce small icons, e.g. for brushing a... and reordering axes $\mathbb{B}^{-}$. Subsequently, by connecting the text-based instructions to the respective visualization, the interactive legend serves as direct linking.

\subsection{Scrollytelling Tutorial}

Scrollytelling [2] is an effective and powerful narrative format to package and transmit complex information [40,44]. The content and organization (visual cues and assignment to one of the three sections) are the same as in the step-by-step guide, but the visual presentation of this method differs. Based on the principles of construction by Nolan et al. [33], text elements can be displayed incrementally by scrolling up and down the screen [40, pp. 95]. Our prototype shows $\mathbf{D}$ the interactive visualization with visual cues (annotations) on the left, an $\mathbf{A}$ introductory sentence, and $\mathbf{C}$ ten instruction steps on the right side of the user interface, see Figure 1.

\subsection{Video Tutorial}

Videos are commonly used in the context of onboarding and help systems [3, 18, 25, 26, 34, 36, 41]. Our video tutorial is based on a guided introduction to parallel coordinates plots and relies on passive instructions, as interaction with the visualization is not supported. Similar to the explanation and structure of the step-bystep guide, we show each mark and interaction element. Moreover, we use a voice-over to describe each step of the explanation. To enable auditory impaired people to understand the explanations, we support textual description with subtitles.

\subsection{Implementation}

The prototypes are implemented using web technologies such as JavaScript, HTML, and CSS. We rely on React [37] as the basic frontend framework to provide a responsive user interface and a fast application and both Vega-Lite [42] and the Vega-Lite wrapper for React [38] to render the charts. For the parallel coordinates plots, we integrated a modified version of the parcoords library [10]. Due to the limitations of interactive features in Vega/Vega-Lite, we enrich the parallel coordinates and other visualizations with in-place annotation using D3.js [11].

\section{EVALUATION}

Our study aimed to investigate (1) for which visualization type (bar chart, horizon graph, change matrix, and parallel coordinates plot) an onboarding is needed by assessing interactions (e.g., click events) with the step-by-step onboarding method and qualitative feedback (see Section 4.1). (2) We investigated differences in the perception and rating of three onboarding methods (interactive step-by-step guide, scrollytelling, and video tutorial) based on parallel coordinates, described in Section 4.2.

\subsection{Visualization Types}

Experiments with MTurk workers were conducted in 2019-2020 to investigate whether and how participants use and reflect on a step-by-step guide as an onboarding method for four visualization types (bar chart, horizon graph, change matrix, and parallel coordinates plot). We chose these four visualization types as we did not intend to exclusively evaluate onboarding for one single visualization type but rather compare different visualization types with diverging levels of familiarity and complexity [6-8, 16, 17, 35].

Methods \& Participants: We conducted the experiments using LimeSurvey [29] and MTurk for deployment. We asked the participants about their level of experience per visualization type. In line with previous work [7], the bar chart shows the highest average level of experience (71.68\%), followed by the horizon graph 
(34.04\%), the parallel coordinates plot (27.19\%), and the change matrix (18.81\%). As the quantitative data of answer correctness and response time no longer provided in-depth information about the usage of visualization onboarding, we concentrate on the qualitative aspects of the study. To qualitatively capture the needs for visualization onboarding, we employed 58 workers $(m=28, f=19$, prefer not to say=1; Age: $M=39.91, S D=9.56$ ) from the crowdsourcing platform. We used the Hotjar behavior analytics software [20] to record and analyze mouse movements and click events. We relied on easy-to-understand time series datasets: weather [14], car data [12], and Olympic medal allocations [28].

Results from Observations and Feedback: Based on observations from the behavioral mouse tracking tool and the qualitative feedback on the step-by-step guide, we identified that for business charts such as the bar chart, the change matrix, and the horizon graph, onboarding was not needed as the charts were "easy to understand" (Bc2, Bc5, Bc36, $\mathrm{Hg} 13, \mathrm{Hg} 43, \mathrm{M} 45)$ and "straightforward" (Bc22). Hence, hardly any mouse movements were recorded. Moreover, participants appreciated the "calming color scheme" (P35) and the coloring of text elements (Bc41, Hg43, Hg45, M26), which is in line with our consideration of using specifications of VegaLite (visual properties) for emphasizing relevant information. For the more complex visualization type (parallel coordinates plot), however, more mouse movement and click events were registered within B our interactive visualization and D step-by-step guide pane. This can be traced back to brushing and re-ordering axes in $\mathbf{B}$ as well as selecting the respective text elements in $\mathbf{D}$ to get familiar with them. Besides, participants claimed that the step-by-step guide leads to a cluttered view (St25, St38), which means that alternative onboarding methods have to be considered.

\subsection{Onboarding Methods}

In our next validation step, we performed further experiments with MTurk workers to determine differences in the qualitative feedback between onboarding methods (scrollytelling and video tutorial) for the parallel coordinates plot.

Methods \& Participants: Similar to the approach described in Section 4.1, we conducted a between-subject design with further 68 MTurk workers $(\mathrm{m}=44, \mathrm{f}=23$, prefer not to say=1; Age: $M=38.11, S D=10.25$ ), using either a step-by-step guide, a scrollytelling, or a video tutorial by assessing their feedback. As Hotjar builds heatmaps on top of screenshots, we were not able to assess the scrolling behavior and thus did not assess mouse movements for the method comparison. Additionally, we performed a sentiment analysis on the qualitative feedback of the three onboarding methods using the sentiment analyser by MonkeyLearn [31]-a machine learning platform that aims to retrieve and classify text information-while also evaluating each review ourselves.

Results from the Feedback: Overall, results show that $83.8 \%$ of the responses can be classified as positive and $16.2 \%$ as negative. We omitted the neutral feedback (e.g., "na", no comment) due to the lack of information. Participants decisively appreciated the condensed, structured, and grouped explanation steps of each of the approaches. On closer examination, the highest positive feedback was given for the video tutorial (93.33\%), followed by the step-by-step guide (81.25\%), and the scrollytelling tutorial $(72.73 \%)$.
Noteworthy is that participants highlighted learning new features during the video tutorial (V26, V42, V50), e.g., "The video was helpful and showed me some features that I wasn't familiar with [..]" (V50). More specifically, "[..] I liked knowing that I could move columns next to one another" (V60), which relates to the re-arrangement of axes. However, the automatically generated voice-over was described as unattractive as it sounded robotic (V3, V65). In contrast, the step-by-step guide did not support the usage and understanding of interactive elements (e.g., filtering and moving axes) or the interpretation of correlations as St34 described: "I have trouble with correlations, but I don't think that is the fault of the guide-although examples would be good" (St34). Regarding the scrollytelling tutorial, users "[..] enjoy that the images on the left do not appear until I need to see them, which prevents confusion" (St38). Hence, the display of information on-demand through scrolling and thereby enriching the visualization type with information increased the perceived satisfaction of the participants.

\section{INITIAL REFLECTIONS ON VISUALIZATION ONBOARDING}

Reflecting on the feedback from our surveys, we summarize the most important insights. We realized that independently of the visualization type and method applied, an easy-to-understand dataset and concrete examples on how to read the chart, support, and increase comprehension are vital. Furthermore, qualitative feedback indicated that the to-the-point descriptions make it easier to absorb information (see Section 4.2). Based on the comments, the competence to learn new interaction techniques can be increased by video tutorials. Likewise, the understanding of introductions is enabled by interactive and linked descriptions of the visualizations $\mathbf{B}$ and the described steps $\mathbf{D}$ (as used for the step-by-step guide and the scrollytelling tutorial). Finally, it is important to consider which visualization types (predominantly unknown and new ones) require an introduction to support the user when needed.

\section{CONCLUSION}

In this paper, we elaborated on the need for visualization onboarding for four different visualization types based on a step-by-step guide. Parallel coordinates plots showed the highest need for onboarding, which is why we additionally investigated the practicability of another two onboarding methods: a scrollytelling and a video tutorial with voice-over. We present the design, implementation, and comparative evaluation using qualitative feedback and mouse tracking of two MTurk studies. Further research is required to determine whether visualization onboarding methods can increase the visualization literacy level of users. Also, it needs to be further investigated whether the presented onboarding concept can be applied to other visualization techniques, such as node-link diagrams, matrices, and more.

Acknowledgments. This work was funded by the Austrian Science Fund as part of the VisOnFire project (FWF P27975-NBL), the Austrian Ministry for Transport, Innovation and Technology (BMVIT) under the ICT of the Future program via the SEVA project (no. 874018), as well as the FFG, Contract No. 881844: "Pro ${ }^{2}$ Future is funded within the Austrian COMET Program Competence Centers for Excellent Technologies under the auspices of the Austrian Federal Ministry for Climate Action, Environment, Energy, Mobility, Innovation and Technology, the Austrian Federal Ministry for Digital and Economic Affairs and of the Provinces of Upper Austria and Styria. COMET is managed by the Austrian Research Promotion Agency FFG." 


\section{REFERENCES}

[1] Basak Alper, Nathalie Henry Riche, Fanny Chevalier, Jeremy Boy, and Metin Sezgin. 2017. Visualization Literacy at Elementary School. In Proc. of CHI (Denver, Colorado, USA) (CHI '17). Association for Computing Machinery, New York, NY, USA, 5485-5497. https://doi.org/10.1145/3025453.3025877

[2] Lorenzo Amabili. 2019. From Storytelling To Scrollytelling: A Short Introduction and Beyond. https://medium.com/nightingale/from-storytelling-to-scrollytellinga-short-introduction-and-beyond-fbda32066964. Accessed: 2021-02-15.

[3] Nikola Banovic, Tovi Grossman, Justin Matejka, and George Fitzmaurice. 2012 Waken: Reverse Engineering Usage Information and Interface Structure from Software Videos. In Proc. of UIST (UIST '12). ACM, New York, NY, USA, 83-92. https://doi.org/10.1145/2380116.2380129

[4] F. Bishop, J. Zagermann, U. Pfeil, G. Sanderson, H. Reiterer, and U. Hinrichs. 2020 Construct-A-Vis: Exploring the Free-Form Visualization Processes of Children TVCG 26, 1 (2020), 451-460. https://doi.org/10.1109/TVCG.2019.2934804

[5] Michelle A. Borkin, Zoya Bylinskii, Nam Wook Kim, Constance May Bainbridge, Chelsea S. Yeh, Daniel Borkin, Hanspeter Pfister, and Aude Oliva. 2016. Beyond Memorability: Visualization Recognition and Recall. TVCG 22, 1 (2016), 519-528. https://doi.org/10.1109/TVCG.2015.2467732

[6] M. A. Borkin, A. A. Vo, Z. Bylinskii, P. Isola, S. Sunkavalli, A. Oliva, and H Pfister. 2013. What Makes a Visualization Memorable? TVCG 19, 12 (Dec 2013), 2306-2315. https://doi.org/10.1109/TVCG.2013.234

[7] Katy Börner, Adam Maltese, Russell Nelson Balliet, and Joe Heimlich. 2016. In vestigating Aspects of Data Visualization Literacy Using 20 Information Visualizations and 273 Science Museum Visitors. Information Visualization 15, 3 (2016), 198-213. https://doi.org/10.1177/1473871615594652

[8] J. Boy, R. A. Rensink, E. Bertini, and J. D. Fekete. 2014. A Principled Way of Assessing Visualization Literacy. TVCG 20, 12 (2014), 1963-1972. https://doi. org/10.1109/TVCG.2014.2346984

[9] Stuart K. Card, Jock D. Mackinlay, and Ben Shneiderman. 1999. Readings in Information Visualization: Using Vision to Think. Morgan Kaufmann Publishers Inc., San Francisco, California.

[10] Kai Chang. 2021. Parallel Coordinates. http://syntagmatic.github.io/parallelcoordinates/ Accessed: 2021-02-18.

[11] D3 Language. 2019. D3 Language. https://d3js.org/. https://d3js.org/ Accessed: 2019-11-20.

[12] Car data. 2020. Car data. http://parallelcoordinates.de/paco/. Accessed: 2020-0420.

[13] Dirk De Bock, Johan Deprez, Wim Van Dooren, Michel Roelens, and Lieven Verschaffel. 2011. Abstract or Concrete Examples in Learning Mathematics? A Replication and Elaboration of Kaminski, Sloutsky, and Heckler's Study. fournal for Research in Mathematics Education 42, 2 (2011), 109-126

[14] ECAD. 2020. ECAD. http://www.ecad.eu. Accessed: 2020-04-20.

[15] Eric G. Freedman and Priti Shah. 2002. Toward a Model of Knowledge-Based Graph Comprehension. In Diagrammatic Representation and Inference (Lecture Notes in Computer Science), Mary Hegarty, Bernd Meyer, and N. Hari Narayanan (Eds.). Springer, Berlin, Heidelberg, 18-30. https://doi.org/10.1007/3-540-460373_3

[16] Mirta Galesic and Rocio Garcia-Retamero. 2011. Graph Literacy: A Cross-Cultural Comparison. Medical Decision Making 31, 3 (2011), 444-457. https://doi.org/10. 1177/0272989X10373805 Publisher: SAGE PublicationsSage CA: Los Angeles, CA

[17] L. Grammel, M. Tory, and M. Storey. 2010. How Information Visualization Novices Construct Visualizations. TVCG 16, 6 (Nov 2010), 943-952. https: //doi.org/10.1109/TVCG.2010.164

[18] Tovi Grossman and George Fitzmaurice. 2010. ToolClips: An Investigation of Contextual Video Assistance for Functionality Understanding. In Proceedings of the SIGCHI Conference on Human Factors in Computing Systems (Atlanta, Georgia, USA) (CHI '10). Association for Computing Machinery, New York, NY, USA 1515-1524. https://doi.org/10.1145/1753326.1753552

[19] Yan Holtz and Conor Healy. 2021. From data to Viz | Find the graphic you need. data-to-viz.com Library Catalog: www.data-to-viz.com.

[20] Hotjar. 2020. Hotjar. https://www.hotjar.com/. Accessed: 2021-02-15.

[21] Samuel Huron, Sheelagh Carpendale, Alice Thudt, Anthony Tang, and Michael Mauerer. 2014. Constructive Visualization. In Proceedings of the 2014 Conference on Designing Interactive Systems (Vancouver, BC, Canada) (DIS '14). Association for Computing Machinery, New York, NY, USA, 433-442. https://doi.org/10. $1145 / 2598510.2598566$

[22] S. Huron, Y. Jansen, and S. Carpendale. 2014. Constructing Visual Representations: Investigating the Use of Tangible Tokens. TVCG 20, 12 (2014), 2102-2111. https: //doi.org/10.1109/TVCG.2014.2346292

[23] Jennifer A. Kaminski, Vladimir M. Sloutsky, and Andrew F. Heckler. 2008. The Advantage of Abstract Examples in Learning Math. Science 320, 5875 (2008), 454-455. https://doi.org/10.1126/science. 1154659

[24] Stephen M. Kosslyn. 1989. Understanding charts and graphs. Applied Cognitive Psychology 3, 3 (1989), 185-225. https://doi.org/10.1002/acp.2350030302_eprint: https://onlinelibrary.wiley.com/doi/pdf/10.1002/acp.2350030302.
[25] Bum Chul Kwon and Bongshin Lee. 2016. A Comparative Evaluation on Online Learning Approaches using Parallel Coordinate Visualization. In Proc. of CHI (CHI '16). ACM Press, New York, NY, USA, 993-997. https://doi.org/10.1145/ 2858036.2858101

[26] Benjamin Lafreniere, Tovi Grossman, and George Fitzmaurice. 2013. Community Enhanced Tutorials: Improving Tutorials with Multiple Demonstrations. Association for Computing Machinery, New York, NY, USA, 1779-1788. https://doi.org/10. $1145 / 2470654.2466235$

[27] Sukwon Lee, Sung-Hee Kim, Ya-Hsin Hung, Heidi Lam, Youn-Ah Kang, and Ji Soo Yi. 2016. How do People Make Sense of Unfamiliar Visualizations?: A Grounded Model of Novice's Information Visualization Sensemaking. TVCG 22, 1 (2016), 499-508. https://doi.org/10.1109/TVCG.2015.2467195

[28] Olympic World Library. 2020. Olympic World Library. https://ibrary.olympic. org/. Accessed: 2020-04-20.

[29] LimeSurvey. 2020. LimeSurvey. http://www.limesurvey.org/. Accessed: 2021-0215.

[30] Susie Lu. 2019. D3 Language. https://d3-annotation.susielu.com/. https://d3annotation.susielu.com/ Accessed: 2019-11-20.

[31] Alex MacCaw, Stephen Blum, Rand Fishkin, and Guillaume Cabane. 2021. Text Analysis. https://monkeylearn.com/ Accessed: 2021-02-18.

[32] Tamara Munzner. 2009. A Nested Process Model for Visualization Design and Validation. TVCG 15, 6 (2009), 921-928. https://doi.org/10.1109/TVCG.2009.111

[33] Deborah Nolan and Jamis Perrett. 2016. Teaching and Learning Data Visualization: Ideas and Assignments. The American Statistician 70, 3 (2016), 260-269. https: //doi.org/10.1080/00031305.2015.1123651

[34] Oluwakemi Ola and Kamran Sedig. 2017. Health Literacy for the General Public: Making a Case for Non-Trivial Visualizations. Informatics 4, 4 (2017), 33. https: //doi.org/10.3390/informatics4040033

[35] Lisa Maria Perkhofer, Peter Hofer, Conny Walchshofer, Thomas Plank, and Hans-Christian Jetter. 2019. Interactive visualization of big data in the field of accounting: A survey of current practice and potential barriers for adoption. Fournal of Applied Accounting Research 20, 4 (Dec. 2019), 497-525. https: //doi.org/10.1108/JAAR-10-2017-0114

[36] Suporn Pongnumkul, Mira Dontcheva, Wilmot Li, Jue Wang, Lubomir Bourdev, Shai Avidan, and Michael F. Cohen. 2011. Pause-and-Play: Automatically Linking Screencast Video Tutorials with Applications. In Proc. of UIST (Santa Barbara, California, USA) (UIST '11). Association for Computing Machinery, New York, NY, USA, 135-144. https://doi.org/10.1145/2047196.2047213

[37] React Language. 2019. React Language. https://reactjs.org/. https://reactjs.org/ Accessed: 2019-11-20.

[38] React Vega. 2019. React Vega. https://github.com/vega/react-vega. https: //github.com/vega/react-vega Accessed: 2019-11-20.

[39] Severino Ribecca. 2020. The Data Visualisation Catalogue. https:// datavizcatalogue.com/. https://datavizcatalogue.com/ Accessed: 2020-03-12.

[40] Nathalie Henry Riche, Christophe Hurter, Nicholas Diakopoulos, and Sheelagh Carpendale. 2018. Data-Driven Storytelling. A K Peters/CRC Press, Boca Raton. https://doi.org/10.1201/9781315281575

[41] P. Ruchikachorn and K. Mueller. 2015. Learning Visualizations by Analogy: Promoting Visual Literacy through Visualization Morphing. TVCG 21, 9 (2015), 1028-1044. https://doi.org/10.1109/TVCG.2015.2413786

[42] A. Satyanarayan, D. Moritz, K. Wongsuphasawat, and J. Heer. 2017. Vega-Lite: A Grammar of Interactive Graphics. TVCG 23, 1 (Jan 2017), 341-350. https: //doi.org/10.1109/TVCG.2016.2599030

[43] A. Satyanarayan, R. Russell, J. Hoffswell, and J. Heer. 2016. Reactive Vega: A Streaming Dataflow Architecture for Declarative Interactive Visualization. IEEE Transactions on Visualization and Computer Graphics 22, 1 (Jan 2016), 659-668. https://doi.org/10.1109/TVCG.2015.2467091

[44] Edward Segel and Jeffrey Heer. 2010. Narrative Visualization: Telling Stories with Data. TVCG 16, 6 (2010), 1139-1148. https://doi.org/10.1109/TVCG.2010.179

[45] Priti Shah and James Hoeffner. 2002. Review of graph comprehension research: Implications for instruction. Educational Psychology Review 14 (2002), 47-69. Issue 1. https://doi.org/10.1023/A:1013180410169

[46] Christina Stoiber, Florian Grassinger, Margit Pohl, Holger Stitz, Marc Streit, and Wolfgang Aigner. 2019. Visualization Onboarding: Learning How to Read and Use Visualizations. preprint. Open Science Framework. https://doi.org/10.31219/osf. io/c38ab

[47] John Swabish and Severinno Ribecca. 2014. The Graphic Continuum: A Poster Project for Your Office. https://policyviz.com/2014/09/09/graphic-continuum/. https://policyviz.com/2014/09/09/graphic-continuum/ Accessed: 2020-03-25.

[48] Yuzuru Tanahashi, Nick Leaf, and Kwan-Liu Ma. 2016. A Study On Designing Effective Introductory Materials for Information Visualization. Computer Graphics Forum 35, 7 (2016), 117-126. https://doi.org/10.1111/cgf.13009

[49] Jarke van Wijk. 2006. Views on Visualization. TVCG 12, 4 (2006), 421-432. https://doi.org/10.1109/TVCG.2006.80

[50] Qiyu Zhi, Alvitta Ottley, and Ronald A. Metoyer. 2019. Linking and Layout: Exploring the Integration of Text and Visualization in Storytelling. Comput. Graph. Forum 38 (2019), 675-685. 